\title{
Impact of case type, length of stay, institution type, and comorbidities on Medicare diagnosis-related group reimbursement for adult spinal deformity surgery
}

\author{
Pierce D. Nunley, MD, ${ }^{1}$ Gregory M. Mundis Jr., MD, ${ }^{2,3}$ Richard G. Fessler, MD, ${ }^{4}$ Paul Park, MD, ${ }^{5}$ \\ Joseph M. Zavatsky, MD, ${ }^{6}$ Juan S. Uribe, MD, ${ }^{7}$ Robert K. Eastlack, MD, ${ }^{3}$ Dean Chou, MD, ${ }^{8}$ \\ Michael Y. Wang, MD, ${ }^{9}$ Neel Anand, MD, ${ }^{10}$ Kelly A. Frank, MS, ${ }^{1}$ Marcus B. Stone, PhD, ${ }^{1}$ \\ Adam S. Kanter, MD, ${ }^{11}$ Christopher I. Shaffrey, MD, ${ }^{12}$ Praveen V. Mummaneni, MD, ${ }^{8}$ and \\ the International Spine Study Group \\ ${ }^{1}$ Spine Institute of Louisiana, Shreveport, Louisiana; ${ }^{2}$ San Diego Center for Spinal Disorders, San Diego, California; ${ }^{3}$ Scripps \\ Clinic, La Jolla, California; ${ }^{4}$ Rush University, Chicago, Illinois; ${ }^{5}$ University of Michigan, Ann Arbor, Michigan; ${ }^{6}$ Spine \& Scoliosis \\ Specialists, Tampa, Florida; ${ }^{2}$ University of South Florida, Tampa, Florida; ${ }^{8}$ University of California, San Francisco, California; \\ University of Miami, Florida; ${ }^{10}$ Cedars-Sinai Medical Center, Los Angeles, California; ${ }^{11}$ University of Pittsburgh Medical Center, \\ Pittsburgh, Pennsylvania; and ${ }^{12}$ University of Virginia Neurological Surgery, Charlottesville, Virginia
}

OBJECTIVE The aim of this study was to educate medical professionals about potential financial impacts of improper diagnosis-related group (DRG) coding in adult spinal deformity (ASD) surgery.

METHODS Medicare's Inpatient Prospective Payment System PC Pricer database was used to collect 2015 reimbursement data for ASD procedures from 12 hospitals. Case type, hospital type/location, number of operative levels, proper coding, length of stay, and complications/comorbidities (CCs) were analyzed for effects on reimbursement. DRGs were used to categorize cases into 3 types: 1) anterior or posterior only fusion, 2) anterior fusion with posterior percutaneous fixation with no dorsal fusion, and 3) combined anterior and posterior fixation and fusion.

RESULTS Pooling institutions, cases were reimbursed the same for single-level and multilevel ASD surgery. Longer stay, from 3 to 8 days, resulted in an additional $\$ 1400$ per stay. Posterior fusion was an additional $\$ 6588$, while CCs increased reimbursement by approximately $\$ 13,000$. Academic institutions received higher reimbursement than private institutions, i.e., approximately $\$ 14,000$ (Case Types 1 and 2) and approximately $\$ 16,000$ (Case Type 3). Urban institutions received higher reimbursement than suburban institutions, i.e., approximately $\$ 3000$ (Case Types 1 and 2) and approximately $\$ 3500$ (Case Type 3). Longer stay, from 3 to 8 days, increased reimbursement between $\$ 208$ and $\$ 494$ for private institutions and between $\$ 1397$ and $\$ 1879$ for academic institutions per stay.

CONCLUSIONS Reimbursement is based on many factors not controlled by surgeons or hospitals, but proper DRG coding can significantly impact the financial health of hospitals and availability of quality patient care.

https://thejns.org/doi/abs/10.3171/2017.7.FOCUS17278

KEY WORDS Medicare; adult spinal deformity; diagnosis-related group; DRG; prospective payment system; spine surgery; reimbursement

$\mathrm{F}$ OR hospitals to continue to provide excellent care for adult spinal deformity (ASD) surgery, they must be able to adequately recover their costs for these procedures. The introduction of Medicare's Prospective Payment System (PPS) in 1983 led to the development of a fixed payment amount for each patient upon hospital discharge (https://www.cms.gov/Medicare/Medicare-Feefor-Service-Payment/AcuteInpatientPPS/index.html). ${ }^{1}$ The fixed payment amount is determined based on the particular diagnosis-related group (DRG) coded at patient dis-

ABBREVIATIONS ASD = adult spinal deformity; $C C=$ complication/comorbidity; $C F R=$ Code of Federal Regulations; $D R G=$ diagnosis-related group; $P$ PS = Prospective Payment System. 
TABLE 1. Case type, DRG code, and description

\begin{tabular}{lcl}
\hline \multicolumn{1}{c}{ Case Type } & DRG Code & \multicolumn{1}{c}{ DRG Description } \\
\hline $1 \& 2$ & 458 & Spinal fusion except cervical w/ spinal curvature or malignancy or infection or extensive fusions w/o CC/major CC \\
\hline $1 \& 2$ w/ CCs & 457 & Spinal fusion except cervical w/ spinal curvature or malignancy or infection or extensive fusions w/ CC \\
\hline $1 \& 2$ w/o deformity & 460 & Spinal fusion except cervical w/o major CC \\
\hline 3 & 455 & Combined anterior/posterior spinal fusion w/o CC/major CC \\
\hline 3 w/ CCs & 454 & Combined anterior/posterior spinal fusion w/ CC \\
\hline
\end{tabular}

charge and it is intended that this fixed amount covers the cost of a typical patient receiving the specific treatment/ surgery designated by the DRG.

However, the value of the reimbursement for the same DRG code differs between institutions based on multiple factors, including institution type (academic or private), institution location (urban or suburban), cost outliers, and disproportionate payments. ${ }^{1}$ Academic institutions are considered to have indirect medical education costs that include salaries of residents/fellows and extra tests/procedures for teaching purposes. This increases the DRG reimbursement rate based on a percentage determined by the ratio of interns and residents to hospital beds (42 Code of Federal Regulations [CFR] Part 412). ${ }^{2}$ Urban and suburban hospitals are reimbursed at different rates based on the application of a wage index. Prevailing wage rates are used in local areas to adjust hospital DRGs to accurately compensate for higher- and lower-wage institutions (42 CFR section 412.60). ${ }^{2}$ Cost outliers compensate for cases of extremely high cost (42 CFR sections 412.84, 412.86), and disproportionate share payments allow more reimbursement to hospitals that treat a larger percentage of low-income patients (42 CFR section 412 ). ${ }^{2}$ A recent study by Ugiliweneza et al. noted that large variation existed within the same DRG codes, although their analysis focused on 30-, 60- and 90-day bundled payments. ${ }^{3}$ Wright et al. also found that cost variations within spinal DRGs are significantly higher than in the total joint replacement DRGs. ${ }^{4}$

Because the DRG coding limits the reimbursement amount available per case based on many factors that are not controllable by the surgeons or hospital, it becomes critical that DRG coding is completed properly for maximum reimbursement. With limited literature, the impact of improper DRG coding to the financial health of hospitals, and ultimately to the availability and quality of patient care, is not well understood. Spine surgeons and hospitals must begin to understand the differences and impacts of DRG coding within their specialty given that inadequate reimbursement may make ASD surgery cost prohibitive. This analysis compiles recent DRG reimbursement data and stratifies it to educate surgeons and hospitals to the reimbursement differences based on proper DRG coding.

\section{Methods}

The Inpatient PPS PC Pricer (cms.gov) was used to collect Medicare reimbursement data from 2015 for ASD procedure DRGs at 12 hospitals across the US. The hospitals included both academic and private institutions in both urban and suburban areas. Academic, or teach- ing, hospitals are defined by 42 CFR Part 403.902 as any institution that received a payment under 1886(d)(5)(B), 1886(h), or 1886(s) of the Social Security Act during the last calendar year for which such information is available. Academic hospitals have programs for interns, residents, and fellows approved by the Accreditation Council for Graduate Medical Education, the American Osteopathic Association, the American Dental Association, or the American Podiatric Medical Association, or one that leads to board certification by the American Board of Medical Specialties. The increase in DRG reimbursement rate for academic hospitals is based on a percentage determined by the ratio (r) of interns and residents to hospital beds (42 CFR Part 412). The indirect adjustment teaching factor is:

Adjustment factor $=\mathrm{c} \times\left[(1+\mathrm{r})^{0.405}-1\right]$,

in which $\mathrm{c}$ is a multiplier set by Congress, currently $1.35 .^{2}$

In accordance with 42 CFR Part 412.64, urban areas are classified as metropolitan statistical areas or metropolitan divisions as defined by the Executive Office of Management and Budget. Each case was evaluated for medical complications/comorbidities (CCs). The reimbursement data were collected for ASD procedures, including anterior, posterior, and circumferential single-level and multilevel ASD surgeries. The surgical cases were categorized into 3 case types: 1) anterior or posterior only fusion (including deformity correction); 2) anterior fusion with posterior percutaneous fixation with no dorsal fusion (including deformity correction); and 3) combined anterior and posterior fixation and fusion. Table 1 includes the DRG codes analyzed with the corresponding case type.

The data were analyzed to isolate the reimbursement differences between case types and the effects of CCs, hospital type, hospital location, number of operative levels, proper coding, and length of stay on reimbursement values.

\section{Results}

Data were categorized and analyzed first using the average DRG for all institutions, followed by institution type (academic vs private) and institution location (suburban vs urban).

\section{All Institutions}

The average DRG reimbursement from all institutions was not affected by the number of fused levels. Case Types 1,2 , and 3 were all reimbursed the same for single-level compared with multilevel fusion. The difference between a 3-day and 8-day stay for all case types was approximately $\$ 1400$. Case Types 1 and 2 are reimbursed the same, an average of $\$ 41,404$ for a 3-day stay with DRG 458. The 
TABLE 2. Comparison of Medicare reimbursements for 3-day and 8-day hospital stay

\begin{tabular}{cccc}
\hline & \multicolumn{2}{c}{ Medicare } & \\
\cline { 2 - 3 } Case Type & $\begin{array}{c}\text { 3-Day } \\
\text { Stay }\end{array}$ & $\begin{array}{c}\text { 8-Day } \\
\text { Stay }\end{array}$ & $\begin{array}{c}\text { \$ Increase } \\
\text { From } \\
\text { 3 to 8 Days }\end{array}$ \\
\hline Case Types 1 \& 2 (DRG 458) & 41,404 & 42,808 & 1404 \\
\hline w/ CC (DRG 457) & 54,476 & 55,881 & 1405 \\
\hline $\begin{array}{c}\text { Case Types 1 \& 2 not coded } \\
\text { as deformity (DRG 460) }\end{array}$ & 31,635 & 33,040 & 1405 \\
\hline Case Type 3 (DRG 455) & 47,992 & 49,397 & 1405 \\
\hline w/ CC (DRG 454) & 61,806 & 63,212 & 1406 \\
\hline
\end{tabular}

addition of posterior fixation without fusion in Case Type 2 compared with Case Type 1, does not result in any additional reimbursement. If a CC is included for Case Types 1 or 2 (DRG 457), the reimbursement is increased to $\$ 54,476$ for a 3-day stay. DRG 457 and 458 include a deformity classification. However, if deformity is not present and/or not charged using DRG 460, reimbursement is $\$ 31,635$ for a 3-day stay. The reimbursement loss is $\$ 9769$ if a deformity case was not coded as deformity. A $360^{\circ}$ fusion, Case Type 3, coded as DRG 455 reimburses $\$ 47,992$ for a 3-day stay. If a CC is present, the reimbursement increases to
$\$ 61,806$. Table 2 summarizes the average of all institutions reimbursement for each case type and stay length.

\section{Reimbursement Differences Between Institution Type and Location}

The highest reimbursement was received at urban/ academic hospitals for all case types (Table 3 ). Categorizing by institution type and location revealed that hospitals classified as academic receive higher reimbursement whether urban or suburban. At private institutions for Case Types $1 \&$ 2, 3-day stays were reimbursed \$28,530 (suburban) and \$31,694 (urban), while at academic institutions reimbursement was $\$ 42,814$ (suburban) and $\$ 46,066$ (urban). The difference is most pronounced between private suburban and urban/academic at more than $\$ 17,000$. When comparing across groups, the difference between urban versus suburban hospitals is approximately $\$ 3000$, while the difference between private versus academic is approximately $\$ 14,000$. For this same procedure (Case Type 1 or 2), if a CC is coded the difference between suburban private and academic urban is more than $\$ 22,000$. The difference between academic versus private remains more pronounced, approximately $\$ 18,000$, compared with approximately $\$ 4000$ for urban versus suburban. Case Type 3 is the highest reimbursed code at all institutions, at $\$ 33,287$ (suburban-private), $\$ 36,841$ (urban-private), $\$ 49,539$ (suburban-academic), and \$53,344 (urban/aca-

TABLE 3. Comparison of Medicare reimbursements between institution type and location

\begin{tabular}{|c|c|c|c|c|c|c|}
\hline \multirow[b]{2}{*}{ Case Type } & \multicolumn{2}{|c|}{ 3-Day Stay } & \multirow[b]{2}{*}{ Difference } & \multicolumn{2}{|c|}{ 8-Day Stay } & \multirow[b]{2}{*}{ Difference } \\
\hline & Private & Academic & & Private & Academic & \\
\hline \multicolumn{7}{|l|}{ Case Types 1 \& 2 (DRG 458) } \\
\hline Urban & 31,694 & 46,066 & 14,372 & 32,188 & 47,945 & 15,757 \\
\hline Suburban & 28,530 & 42,814 & 14,284 & 28,738 & 44,211 & 15,473 \\
\hline Difference & 3164 & 3253 & & 3450 & 3735 & \\
\hline \multicolumn{7}{|l|}{ w/ CC (DRG 457)* } \\
\hline Urban & 41,906 & 60,508 & 18,602 & 42,400 & 62,388 & 19,988 \\
\hline Suburban & 37,968 & 56,158 & 18,190 & 38,176 & 57,555 & 19,379 \\
\hline Difference & 3938 & 4350 & & 4224 & 4833 & \\
\hline \multicolumn{7}{|c|}{ Case Types $1 \& 2$ not coded as deformity (DRG 460)† } \\
\hline Urban & 24,063 & 35,274 & 11,211 & 24,557 & 37,154 & 12,597 \\
\hline Suburban & 21,479 & 32,841 & 11,362 & 21,686 & 34,238 & 12,552 \\
\hline Difference & 2585 & 2433 & & 2871 & 2916 & \\
\hline \multicolumn{7}{|l|}{ Case Type 3 (DRG 455) $\ddagger$} \\
\hline Urban & 36,841 & 53,344 & 16,503 & 37,335 & 55,224 & 17,889 \\
\hline Suburban & 33,287 & 49,539 & 16,252 & 33,495 & 50,936 & 17,441 \\
\hline Difference & 3555 & 3806 & & 3841 & 4289 & \\
\hline \multicolumn{7}{|l|}{ w/ CC (DRG 454)* } \\
\hline Urban & 47,632 & 68,606 & 20,974 & 48,126 & 70,486 & 22,360 \\
\hline Suburban & 43,260 & 63,642 & 20,382 & 43,468 & 65,039 & 21,571 \\
\hline Difference & 4372 & 4965 & & 4658 & 5448 & \\
\hline
\end{tabular}

All data given in US dollars

* Any case type that includes a CC that is not properly coded results in losses between $\$ 9438$ and $\$ 15,262$.

† Case Types 1 \& 2 incorrectly coded without deformity when a deformity exists, result in losses between $\$ 7051$ and $\$ 10,792$.

$\ddagger$ Case Type 3 is independent of deformity, but the addition of posterior fusion results in increased reimbursement between $\$ 4757$ and $\$ 7279$. 
demic). When Case Type 3 includes CC, the reimbursement difference is more than $\$ 25,000$ between suburbanprivate and urban-academic.

Following similar trends as above, academic institutions are reimbursed a larger amount when the stay is increased from 3 to 8 days, although the increase for all institutions is minimal. The increase, independent of DRG code, ranges from $\$ 208$ to $\$ 494$ for private institutions and from $\$ 1397$ to $\$ 1879$ for academic institutions.

\section{Reimbursement Differences: Coding and Institution Types and Locations}

Similar to the analysis performed with combined institutions, improper coding would result in reimbursement losses. All cases are reimbursed the same for single-level or multilevel ASD surgery, leaving posterior fusion, CC, and deformity as variables that could change reimbursement. CC for a Case Type 1 or 2 results in increased reimbursement of $\$ 9438$ (suburban) and \$10,212 (urban) for private institutions and $\$ 13,344$ (suburban) and $\$ 14,442$ (urban) for academic institutions, independent of length of stay. For a Case Type 3, the increase for $\mathrm{CC}$ is more pronounced: \$9973 (suburban) and \$10,791 (urban) for private institutions, and $\$ 14,103$ (suburban) and $\$ 15,262$ (urban) for academic institutions.

Similar to CC, if deformity exists, but is not properly coded on a Case Type 1 or 2, all institutions lose reimbursement money. Private institutions lose \$7051 (suburban) and $\$ 7631$ (urban), while academics institutions lose $\$ 9973$ (suburban) and \$10,792 (urban), independent of length of stay.

\section{Discussion}

The results from our analysis of the PPS DRG codes related to ASD fusion in 2015 confirm that the DRG code drastically impacts hospital reimbursement. In addition to the DRG code used, the institution type and location also impact the reimbursement amount.

\section{Case Type, Deformity, and CC}

This study found a similar result to recent publications that reported that there is no difference in reimbursement for additional levels treated. However, increasing complexity does have higher reimbursement, but this value remains variable. ${ }^{3,4}$ Specific to our study, the relationship between Case Type 1 and Case Type 2 is important to understand, as the trend is consistent across all institutions. The reimbursement for Case Types 1 and 2 (DRG 458) indicates that the addition of posterior implants (without a posterior fusion) results in no additional payment to the hospital. Although Case Type 2 involves flipping a patient for posterior access and additional implants compared with Case Type 1, there is no unique DRG code for the Case Type 2 procedure. However, the addition of posterior fusion with a Case Type 2 qualifies the procedure as Case Type 3 and involves a unique DRG (DRG 455). The reimbursement difference from DRG 458 to DRG 455 is between $\$ 4757$ and $\$ 7279$, depending on institution type, location, and length of stay.

Case Types 1 and 2 also need special attention paid to the DRG code when the procedure involves a deformity. If the operative case qualifies as a spinal deformity, yet DRG 460 is used for reimbursement, the loss can range from $\$ 7052$ to $\$ 10,792$, depending on institution type, location, and length of stay. Case Type 3, DRG 455, remains unchanged if a deformity is present.

The presence of a CC impacts all case types across all institutions. The presence of a CC results in a unique DRG code: DRG 457 for Case Types 1 and 2, and DRG 454 for Case Type 3. If a CC occurs and is not coded to the proper DRG, the losses in hospital reimbursement range from $\$ 9438$ to $\$ 15,262$.

\section{Length of Stay}

The length of stay in the hospital following Case Types 1,2 , or 3 are the same DRG codes as described above. It is noteworthy that for any institution type or location, the increase in reimbursement is minimal from a 3-day to an 8-day stay. The range is \$208-\$494 (private) and \$1397-\$1897 (academic) increase for the additional 5 days, an average between $\$ 40$ and $\$ 380$ per day. Wright et al. also found that increasing length of stay did not have an impact on the amount of reimbursement. They noted that this effect is compounded by the lack of differences in reimbursement for multiple levels and minimally invasive surgery versus open surgical procedures. ${ }^{4}$ While it is critical that patient care be the priority in determining the length of stay in the hospital, the financial impacts must be understood, i.e., extending a patient stay in the hospital is not reimbursed well.

\section{Institution Type, Location, and Other Factors}

Academic institutions and institutions located in urban settings receive higher reimbursement than their private and suburban counterparts. This is expected, due to the payment structure created and adopted by Medicare. The DRG coding includes 4 adjustment factors: application of a wage index, indirect medical education costs, cost outliers, and disproportionate share payments. The application of a wage index increases the DRG reimbursement for institutions in an area with a higher prevailing wage, typically urban areas. Indirect medical education costs increase the reimbursement to academic institutions to adjust for the expense of additional tests/procedures performed, the training of medical staff, and the higher ratio of medical staff to patient. The cost outliers account for cases with "extremely high overall costs," based on a limit established by the Centers for Medicare \& Medicaid Services. The disproportionate share payments reimburse additional amounts to institutions that treat a large percentage of lowincome patients. The cost outliers and disproportionate share payments were not analyzed as a part of this study.

Our analysis indicates that academic versus private procedures have differences in reimbursement ranging from $\$ 11,211$ to $\$ 20,974$ (3-day stay) with the academic institutions always receiving higher reimbursement. The increasing range follows the complexity of the case type, with the $\$ 11,211$ difference occurring on a Case Type 1 or 2 without deformity and the $\$ 20,974$ reimbursement difference occurring on a Case Type 3 with CC. 
The urban and suburban reimbursement follows a similar trend to academic and private institutions, although the reimbursement differences are less, with a range from $\$ 2433$ to $\$ 4965$. The range also follows the increasing complexity of the case type, with Case Types 1 and 2 without deformity reimbursing $\$ 2433$ more for urban institutions. A Case Type 3 with CC reimburses $\$ 4965$ more to the urban institution when compared with suburban institution.

\section{Conclusions}

It is clear that some factors (institution type and location) cannot be controlled by the surgeon or hospital when performing ASD surgery for a Medicare patient. Proper understanding and use of DRG codes is within surgeon and hospital control and is critical to ensuring the highest hospital reimbursement for procedures. The financial survival of hospitals is critical to maintaining the best patient care for complex procedures such as ASD fusion. This analysis is the first step in understanding the impact of proper DRG documentation and coding on hospital revenue, which can have a large impact on the sustainability of complex spinal procedures in the future.

\section{References}

1. Gottlober P (ed): Medicare Hospital Prospective Payment System. How DRG Rates are Calculated and Updated. Washington, DC: Department of Health and Human Services, 2001 (https://oig.hhs.gov/oei/reports/oei-09-0000200.pdf) [Accessed October 17, 2017]

2. Prospective Payment Systems for Inpatient Hospital Services, 42 CFR Part 412 (2011)

3. Ugiliweneza B, Kong M, Nosova K, Huang KT, Babu R, Lad SP, et al: Spinal surgery: variations in health care costs and implications for episode-based bundled payments. Spine (Phila Pa 1976) 39:1235-1242, 2014

4. Wright DJ, Mukamel DB, Greenfield S, Bederman SS: Cost variation within spinal fusion payment groups. Spine (Phila Pa 1976) 41:1747-1753, 2016

\section{Disclosures}

Dr. Nunley reports direct stock ownership in Amedica, Paradigm, and Spineology; being a patent holder for K2M and LDR Spine; being a consultant for K2M; and serving on the speaker's bureau for K2M and LDR Spine. Dr. Mundis Jr. reports being a consultant and patent holder for NuVasive and K2M; and receiving travel support from DePuy. Dr. Fessler reports ownership in Queue Innovations. Dr. Park reports being a consultant for Globus,
Medtronic, NuVasive, and Zimmer-Biomet; and receiving royalties from Globus. Dr. Zavatsky reports direct stock ownership in Vivex and Sentio; being a consultant for DePuy Synthes, Stryker, and Zimmer-Biomet; and receiving royalties from ZimmerBiomet. Dr. Uribe reports being a consultant for NuVasive. Dr. Eastlack reports direct stock ownership in Alphatec, NuVasive, and Seaspine; ownership in Spine Innovation; being a consultant for Aesculap, Alphatec, NuVasive, Titan, Seaspine, and K2M; and being a patent holder for Globus, Invuity, NuTech, and Spine Innovation. Dr. Chou reports being a consultant for Medtronic and Globus. Dr. Wang reports being a consultant for DePuySynthes Spine, JoiMax, and K2M; and being a patent holder for Depuy-Synthes Spine. Dr. Anand reports being a consultant for Medtronic and Globus Medical; direct stock ownership in Globus Medical and Medtronic; and being a patent holder for Medtronic. Dr. Frank and Dr. Stone report support of non-study-related clinical or research effort from LDR Spine. Dr. Kanter reports being a consultant for NuVasive, and being a patent holder for ZimmerBiomet. Dr. Shaffrey reports direct stock ownership in NuVasive; and being a consultant and patent holder for Medtronic, NuVasive, and Zimmer-Biomet. Dr. Mummaneni reports being a consultant for DePuy Spine and Stryker Spine; direct stock ownership in Spinicity/ISD; clinical or research support for the study described from ISSG; receiving royalties from DePuy Spine, Thieme publishing, and Springer publishing; receiving honoraria from Globus and AOSpine; and receiving a grant from AOSpine.

\section{Author Contributions}

Conception and design: Nunley, Mundis, Mummaneni. Analysis and interpretation of data: Nunley, Mundis, Frank, Stone. Drafting the article: Nunley, Frank, Stone. Critically revising the article: all authors. Reviewed submitted version of manuscript: all authors. Approved the final version of the manuscript on behalf of all authors: Nunley.

\section{Supplemental Information}

\section{Previous Presentations}

Portions of the work were presented in abstract form at the Proceedings of the CNS, San Diego, California, September 24-28, 2016; Proceedings of the International Meeting on Advanced Spine Techniques, Washington, DC, July 13-16, 2016; Proceedings of the American Academy of Orthopaedics, San Diego, California, March 14-18, 2017; Proceedings of Disorders of the Spine and Peripheral Nerves, March 8-11, 2017; Proceedings of the International Society for the Advancement of Spine Surgery, Boca Raton, Florida, April 12-15, 2017; Proceedings of the Lumbar Spine Research Society, Chicago, Illinois, April 6-7, 2017; and the Proceedings of the North American Spine Society Spine Summit, Park City, Utah, February 22-25, 2017.

\section{Correspondence}

Pierce D. Nunley, Spine Institute of Louisiana, 1500 Line Ave., Ste. 200, Shreveport, LA 71101. email: pnunley@ louisianaspine.org. 